\title{
The Community Pharmacist's Role in Reducing Cardiovascular Risk Factors in Lebanon: A Longitudinal Study
}

\author{
Iqbal M. Fahs ${ }^{\text {a }}$ Souheil Hallit ${ }^{b-g}$ Mohamad K. Rahal ${ }^{\mathrm{a}}$ Diana N. Malaeb \\ ${ }^{a}$ School of Pharmacy, Lebanese International University, Mouseitbah, Lebanon; b Faculty of Pharmacy, Saint-Joseph \\ University of Beirut, Beirut, Lebanon; ' ${ }^{C}$ Lebanese University, Faculty of Pharmacy, Beirut, Lebanon; ${ }^{d}$ Holy Spirit

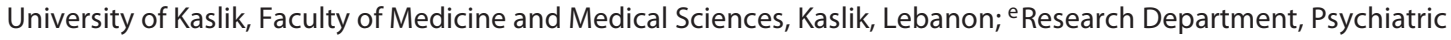 \\ Hospital of the Cross, Jal Eddib, Lebanon; ' Occupational Health Environment Research Team, U1219 BPH Bordeaux \\ Population Health Research Center Inserm, Université de Bordeaux, Bordeaux, France; 9 INSPECT-LB: Institut \\ National de Sante Publique, Epidemiologie Clinique et Toxicologie, Faculty of Public Health, Lebanese University, \\ Beirut, Lebanon
}

\section{Significance of the Study}

- Counseling and education of patients by pharmacists resulted in significant improvements in body mass index, blood pressure, lipid panel levels, and awareness scores, which may manifest as meaningful reduction in cardiovascular risk factors. Our results suggest that the pharmacist can play an important role in preventing and reducing cardiovascular risk factors.

\section{Keywords}

Cardiovascular diseases - Monitoring · Pharmacist ·

Risk factors $\cdot$ Role

\begin{abstract}
Objective: To assess the role of the pharmacist in modifying risk factors for cardiovascular disease (CVD) among Lebanese adults in urban and rural areas. Methods: In a prospective survey, 865 out of 1,000 participants aged $\geq 45$ years, previously interviewed, agreed to be followed at 1 and 2 years time points. Parameters including blood pressure, lipid profile, blood glucose, average number of risk factors,
\end{abstract}

and atherosclerotic CVD (ASCVD) risk were assessed and evaluated at the beginning of the study, then after 1 and 2 years. Results: During both follow-ups, the mean average body mass index and systolic blood pressure decreased significantly and the lipid profile improved significantly. Further significant improvements in ASCVD risk occurred during the second follow-up. Monitoring parameters revealed significant improvements as well. Conclusion: This study showed that a plan that includes pharmacists, who regularly monitor and follow-up patients, could improve CVD prevention through the reduction of risk factors.

(c) 2018 The Author(s)

Published by S. Karger AG, Basel

\begin{tabular}{ll}
\hline KARGER & $\begin{array}{l}\text { Ko 2018 The Author(s) } \\
\text { Published by S. Karger AG, Basel }\end{array}$ \\
$\begin{array}{l}\text { Openger } \\
\text { E-Mail karger@karger.coms }\end{array}$ \\
www.karger.com/mpp & $\begin{array}{l}\text { This is an Open Access article licensed under the Creative Commons } \\
\text { Attribution-NonCommercial-4.0 International License (CC BY-NC) } \\
\text { (http://www.karger.com/Services/OpenAccessLicense), applicable to } \\
\text { the online version of the article only. Usage and distribution for } \\
\text { commercial purposes requires written permission. }\end{array}$
\end{tabular}

Souheil Hallit

Street 8, Building 560, 1st floor

Biakout, Mount Lebanon, Beirut (Lebanon)

E-Mail souheilhallit@hotmail.com 


\section{Introduction}

Almost 18 million individuals die annually due to cardiovascular diseases (CVDs), with more than $75 \%$ of cardiovascular deaths occurring in low- and middle-income countries [1]. For instance, ischemic heart disease was the number one killer in Lebanon in 2010, accounting for $32.2 \%$ of overall mortalities [2]. Different modifiable and nonmodifiable CVD risk factors have been identified and studied [1]. CVDs were found to be largely driven by modifiable risk factors such as tobacco use, heavy alcohol intake, physical inactivity, unhealthy diet, obesity, hypertension, diabetes, and dyslipidemia; hence, targeting and modifying these factors can prevent and reduce CVDs [1]. Despite continuous improvements in healthcare in general, and in cardiovascular risk factors management in particular, control of CVDs is far from optimal [3].

Pharmacists, traditionally considered "medication-dispensers," are highly accessible healthcare providers who can play a vital and critical role in the success of modifying CVD risk factors [4]. Pharmacists' interventions can be conducted through education of patients, screening for risk factors and monitoring, and referral to physicians or management of medication [4]. Different studies support this collaborative and patient-centered role of pharmacists [4]. Collaborative practice agreements are important to integrate pharmacists into the health-care teams in order to maximize their impact. Such agreements expand the role of the pharmacists' role in initiating, modifying, discontinuing, and monitoring drug therapy, ordering or administering laboratory tests, and performing limited physical exams such as checking vital signs and assessing edema [5]. In addition, clinical pharmacy services can provide valuable inputs in identifying drug-related problems and providing suggestions on how they might be avoided [6].

Scientific literature demonstrates the effective role of pharmacists in modifying individual CVDs risk factors like smoking [7], diabetes [8], hypertension [9], dyslipidemia [10], and other behavioral risk factors. However, recent guidelines have emphasized the importance of overall assessment of CVD risk and the management of multiple risk factors at the same time [11]. Despite several studies [9, 12$15]$ on the role of the pharmacist in targeting multiple CVD risk factors, this topic has not been extensively studied especially in the Middle East and Northern African region.

In Lebanon, where national studies suggest an alarming trend in elevated CVDs risk factors $[2,16]$, the role of pharmacists in targeting and modifying these factors has not been studied yet. In addition, leading cardiology societies in 2012 advised the reduction of mortality due to noncommu- nicable diseases by $25 \%$ by the year 2025 [17]. This led us to conduct the current study to assess the role of pharmacists in modifying CVD risk factors among Lebanese individuals 45 years of age and above in urban and rural areas.

\section{Methods}

\section{Study Design}

This is a cross-sectional prospective longitudinal follow-up study carried out in Lebanon over a 2 -year period. The study was approved by the Ethical Committee at Lebanese International University. One thousand individuals who had been initially recruited in March 2015 for the prevalence and assessment of awareness of CVD risk factors [16] were followed-up in the current study after 1 and 2 years. In the initial study, the sample was drawn randomly from community pharmacies based on stratified cluster sampling [16]. The strata were the 6 districts of Lebanon and clusters were further selected at the level of districts (urban and rural areas). Ninety-six community pharmacies constituted the primary sampling units and 8 pharmacies were randomly selected from the urban and rural districts using the Research Randomizer computer program [18]. The principal investigators in the study (clinical pharmacists) directly approached and interviewed participants who were enrolled in the study. Individuals 45 years of age and above, who came for normal medications or for para-pharmaceuticals were enrolled in the study. A para-pharmaceutical product is a substance or compound that does not correspond to the legal definition of a medicinal product by virtue of their composition, utilization, or presentation [19].

Individuals who by themselves reported an established CVD, being pregnant or lactating women, cancer patients, and those with mental illnesses were excluded. Out of the 1,000 individuals initially enrolled, 865 participants were followed-up after 1 year on March 2016, with a refusal rate of $13.5 \%$. The refusal to continue into the follow-up phase of the study was mainly due to the fact that those people were not regular customers at the community pharmacy where the data collection was done, which complicated the follow-up process. In March 2017 and after a 2-year period, 841 participants were interviewed with 24 drop-out cases due to loss of contact. The interviewing pharmacists educated the participants after each contact using English and Arabic brochures already used by the American University of Beirut Medical Center as part of increasing awareness about CVDs. The topics addressed dietary and lifestyle changes, hypertension, diabetes, and dyslipidemia monitoring and management $[20,21]$.

\section{Data Collection}

The data collection sheet retrieved information regarding participants' demography, counseling, monitoring, and biochemical parameters. It was established based on validated and standardized questionnaires including World Health Organization STEPwise (WHO STEPS) instrument guideline for noncommunicable diseases [22], Behavioral Risk Factor Surveillance System [23], International Physical Activity questionnaire [24], Food Frequency Questionnaire [25], Adult Questionnaire [26], and Heart Disease Fact Questionnaire [27]. All participants signed written consents prior to enrolment. Data were stripped of any personal information to ensure confidentiality of the patients. 


\section{Measurements}

Parameters, including height, weight, blood pressure, blood glucose, lipid profile, awareness score, average number of elevated CVDs risk factors and ASCVD risk were measured and assessed. Height and weight measurements were done based on the recommendations of the National Health and Nutrition Examination Surveys [28]. Individuals were weighed barefoot using an electronic scale (LAICA ${ }^{\circledR}$, Mod. PS50090) in light indoor clothing and recorded to the nearest $0.1 \mathrm{~kg}$. Height was measured without shoes using portable stadiometer (Seca $\left.{ }^{\circledR}, 213\right)$ and recorded to the nearest $0.5 \mathrm{~cm}$. Blood pressure was measured based on Joint National Committee guidelines for 3 readings with a digital blood pressure (Omron ${ }^{\circledR}$, M3 IT) machine [29]. Participants were asked to rest for $5 \mathrm{~min}$ between blood pressure measurements and to sit in a back-supported position with the arm at the level of the heart. Tobacco, alcohol, and caffeine were not allowed for at least $120 \mathrm{~min}$ before measurements were taken. An average of the second and the third reading was taken. Fasting blood glucose (FBG) and lipid panel measurements were measured in a clinical laboratory; the relevant clinical pharmacists retrieved the records from the laboratory for evaluation.

Awareness of risk factors for CVDs was computed as a continuous variable. The awareness of CVD risk factors was assessed based on 8 questions from the Heart and Disease Fact Questionnaire [27] retrieving knowledge on smoking, obesity, alcohol consumption, improper diet, physical inactivity, hypertension, diabetes, and dyslipidemia.

Atherosclerotic CVD (ASCVD) risk was calculated using Pooled Cohort Equations for patients with available lipid panel records and age less than or equal to 79 years [30]. The ASCVD risk score is based on age, gender, SBP and treatment status, total cholesterol (TC) level, and HDL level [30]. The projected 10-year risk calculated was further classified into low risk $(\leq 10 \%)$, intermediate risk (10-20\%), and high risk ( $\geq 20 \%)$.

A risk factor at goal evaluation was conducted based on the following criteria: smoking cessation, physical activity most days of the week, consumption of cardio-protective servings of fruits $(\geq 4$ servings/day) and vegetables ( $\geq 5$ servings/day), moderate alcohol consumption (maximum 2/day for men and 1/day for women), BMI below $25 \mathrm{~kg} / \mathrm{m}^{2}$, blood pressure of less than 140/90, TC less than $200 \mathrm{mg} / \mathrm{dL}$, triglycerides (TG) less than $150 \mathrm{mg} / \mathrm{dL}$, low density lipoprotein (LDL) less than $100 \mathrm{mg} / \mathrm{dL}$ and HDL above $60 \mathrm{mg}$ / dL.

\section{Outcomes}

The primary outcome was change in the average number of elevated CVDs risk factors. Secondary outcomes include changes in body mass index (BMI), systolic blood pressure (SBP), FBG, lipid profile, awareness score, and ASCVD risk.

\section{Statistical Analysis}

Data analysis was conducted with the statistical software package "Statistical Package for Social Sciences" software (SPSS, version 21). Mean ( \pm SD) was used to describe continuous variables, while descriptive statistics were used to describe patient characteristics. The studied groups (urban and rural) were compared using the chi-square test for nominal variables, and the analysis of variance test for continuous variables. Mean changes in BMI, SBP, FBG, TG, TC, LDL, HDL, awareness, score, ASCVD risk score and average number of risk factors were assessed for significant modi-
Table 1. Baseline characteristics of the participants

\begin{tabular}{lc}
\hline Parameter & $\begin{array}{l}\text { Total } \\
(\text { mean } \pm \text { SD; } n=865)\end{array}$ \\
\hline Age, years & $55 \pm 9.5$ \\
Gender & \\
$\quad$ Male & $49 \%(427)$ \\
$\quad$ Female & $51 \%(438)$ \\
BMI, kg/m & $28 \pm 4.5$ \\
SBP, mm Hg & $131 \pm 16$ \\
FBG, mg/dL & $103 \pm 25$ \\
TG, mg/dL & $195 \pm 85$ \\
TC, mg/dL & $217 \pm 48$ \\
HDL, mg/dL & $43 \pm 11$ \\
LDL, mg/dL & $138 \pm 49$ \\
Awareness score & $5.7 \pm 1.4$ \\
ASCVD risk & $11 \pm 8$ \\
Average number of risk factors & $2 \pm 1$ \\
\hline
\end{tabular}

FBG, fasting blood glucose; BMI, body mass index; DBP, diastolic blood pressure; HDL, high-density lipoprotein; LDL, lowdensity lipoprotein; $n$, number; TC, total cholesterol; TG, triglycerides; RBG, random blood glucose; SBP, systolic blood pressure; ASCVD, atherosclerotic cardiovascular diseases.

fications after 1 and 2 years using paired sample $t$ test and analysis of variance test. All reported $p$ values were 2 -sided with the alpha set at a significance of 0.05 .

\section{Results}

\section{Baseline Characteristics}

The current study followed-up 865 participants, 427 (49\%) males and 438 (50\%) females, with a mean age of $54 \pm 9.5$ years), who were approached previously in different urban $(n=286)$ and rural $(n=579)$ areas across Lebanon. The baseline characteristics of the participants are summarized in Table 1.

The BMI of the studied population was $28.2 \mathrm{~kg} / \mathrm{m}^{2}$ indicating an overweight population. $42 \%$ were smokers, $2 \%$ drank alcohol heavily, $55 \%$ engaged in moderate activities for at least $10 \mathrm{~min}$ for $\geq 4$ times/week. A low proportion of the participants, 8 and $4 \%$ consumed cardioprotective servings of fruits and vegetables respectively.

SBP was suggestive of pre-hypertension with an average of $131 \mathrm{~mm} \mathrm{Hg}$. Almost two-third of the participants $62 \%$ were hypertensive, $30 \%$ were pre-hypertensive, and only $8 \%$ had normal BP measurements (Fig. 1). FBG was indicative of pre-diabetes with a mean around $102 \mathrm{mg} /$ $\mathrm{dL}$. The baseline averages of the lipid profile revealed borderline high TG, TC, and LDL. A good awareness score 


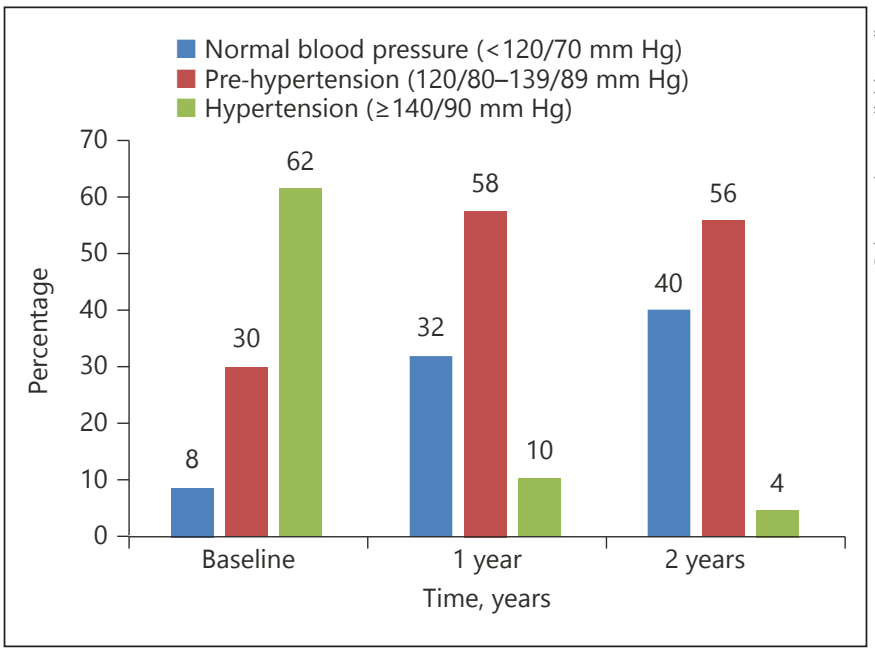

Fig. 1. Blood pressure categories distribution at baseline, 1 and 2 years.

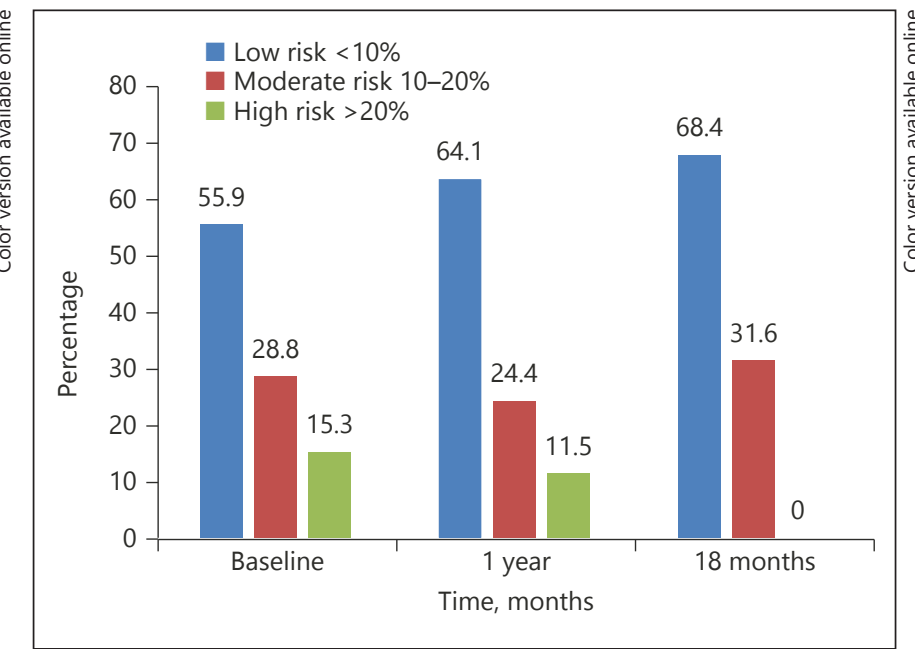

Fig. 2. Categorized ASCVD risk distribution at baseline, at 1 year and 2 years.

Table 2. Parameters that changed significantly between 0,1 and 2 years (paired samples test)

\begin{tabular}{lcccccc}
\hline Variable & $\begin{array}{l}\text { Baseline } \\
\text { value }\end{array}$ & $\begin{array}{l}\text { Value after } \\
1 \text { year }\end{array}$ & $\begin{array}{l}\text { Value after } \\
2 \text { years }\end{array}$ & $\begin{array}{l}p \text { value for } \\
\# 0-1 \text { years }\end{array}$ & $\begin{array}{l}p \text { value for } \\
\# 1-2 \text { years }\end{array}$ & $\begin{array}{l}p \text { value for } \\
\# 0-2 \text { years }\end{array}$ \\
\hline ASCVD risk & $11 \pm 8$ & $9 \pm 7$ & $8 \pm 5$ & 0.01 & 0.01 & 0.01 \\
Average risk factors & $2 \pm 1$ & $1 \pm 0.8$ & $1 \pm 0.7$ & 0.01 & 0.01 & 0.02 \\
BMI, kg/m 2 & $28 \pm 4.5$ & $28 \pm 6$ & $27 \pm 5$ & 0.01 & 0.01 & 0.01 \\
SBP, mm Hg & $131 \pm 16$ & $128 \pm 11$ & $125 \pm 10$ & 0.01 & 0.01 & 0.01 \\
FBG, mg/dL & $103 \pm 25$ & $103 \pm 24$ & $102 \pm 24$ & 0.13 & 0.07 & 0.09 \\
Awareness score & $5.7 \pm 1.4$ & $6.6 \pm 0.8$ & $6.7 \pm 0.6$ & 0.01 & 0.01 & 0.01 \\
TC, mg/dL & $217 \pm 48$ & $217 \pm 39$ & $200 \pm 34$ & 0.01 & 0.01 & 0.02 \\
TG, mg/dL & $195 \pm 85$ & $194 \pm 47$ & $175 \pm 39$ & 0.01 & 0.01 & 0.01 \\
HDL, mg/dL & $43 \pm 11$ & $44 \pm 6$ & $48 \pm 6$ & 0.01 & 0.01 & 0.01 \\
LDL, mg/dL & $138 \pm 49$ & $137 \pm 34$ & $126 \pm 33$ & 0.01 & 0.01 & 0.01 \\
\hline
\end{tabular}

ASCVD, atherosclerotic cardiovascular diseases; BMI, body mass index; SBP, systolic blood pressure; FBG, fasting blood glucose; TC, total cholesterol; TG, triglycerides; HDL, high-density lipoprotein; LDL, low-density lipoprotein.

of around 5.7 out of 8 was reported, with most of the participants having an average of 2 CVD risk factors and a moderate baseline ASCVD risk of 11\%.

\section{Modification of Parameters during the First}

Follow-Up Phase

Paired sample $t$ test (Table 2) for continuous variables revealed many significant changes after 1 and 2 years. Table 2 summarizes the measured parameters after 1 year. Both the mean average BMI and SBP decreased significantly. Consequently, 77\% who had been previously hypertensive became pre-hypertensive and $66 \%$ who had been previously pre-hypertensive had normal BP (Fig. 1). FBG showed no significant improvement after 1 year.
Lipid profiles improved significantly after 1 year; TG, TC and LDL decreased significantly, while high-density lipoprotein increased.

The awareness score, the average number of risk factors, and ASCVD risk also demonstrated significant improvements during the first follow-up into a score of 6.6 out of 8 with an average of 1.3 risk factors per participant and an ASCVD risk of $9 \%$. ASCVD risk was further categorized into low, moderate, and high risk. Figure 2 illustrates categorized ASCVD risk at baseline and during the 2 follow-ups. Initially $29 \%$ of the participants had moderate risk and $15 \%$ had high risk; after the 1-year interval the percentage of those with moderate and high risk decreased significantly to 24 and $12 \%$ respectively. 


\section{Modification of Parameters during the Second}

\section{Follow-Up Phase}

Table 2 summarizes the parameters during the second follow-up. BMI and SBP both significantly decreased further after another 9 months. Consequently, $64 \%$ of hypertensive participants at the first follow-up became pre-hypertensive after 2 years and $20 \%$ of pre-hypertensive participants had normal BP (Fig. 1). On the contrary, FBG levels showed no improvement $(p=0.07)$.

Lipid profiles also showed further significant improvement. TG and TC decreased, while HDL increased. The awareness score, the average number of risk factors, and ASCVD risk improved significantly during the second follow-up into an awareness score of 6.8 with 1.1 average risk factors and around 8\% ASCVD risk. During this phase, the ASCVD risk decreased further with $68 \%$ of participants having low risk and 32\% having moderate risk, while those with high risk became null (Fig. 2).

\section{Discussion}

This is the first study in Lebanon that assessed the role of pharmacists in modifying CVD risk factors. Implemented interventions over 1 and 2 years by pharmacists through the education and counseling of patients resulted in a statistically significant improvement in the primary outcome, which is the average number of CVD risk factors. All secondary outcomes including BMI, SBP, TC, TG, LDL, HDL, awareness score, and ASCVD risk showed significant improvements except for FBG.

The baseline average number of CVD risk factors (around 2) among the study participants significantly decreased after 1 and 2 years. This significant reduction was comparable to that achieved in a study conducted by Bagwell et al. [15] after 6 months of patient education and follow-up by pharmacists. In comparison to our study in which BMI significantly decreased, BMI was not observed to change in the study of Bagwell et al. [15]. This can be explained by the relatively long duration of follow-up in our study of 1 and 2 years compared to 6 months in Bagwell et al. [15]. However, the reduction was not clinically meaningful. Baseline BMI in the present study was similar to those reported previously in Lebanon by Yamout et al. [31], where $40 \%$ were overweight and $30.3 \%$ were obese.

SBP and DBP readings at baseline revealed $62 \%$ of the studied population as hypertensive and $30 \%$ as pre-hypertensive. This result is much higher than that demonstrated in Bahrain by Al-Nooh et al. [32], who reported that $22 \%$ of participants had SBP $\geq 140$ and $23 \%$ had DBP $\geq 90 \mathrm{~mm} \mathrm{Hg}$. However, in our study, mean SBP significantly decreased as in the studies of Bagwell et al. [15] and Tsuyuki et al. [14] after 3 months of follow-up with pharmacist care intervention group and usual care group. As a consequence, the percentage of those with hypertensive readings in our study decreased to $3.9 \%$.

Baseline FBG levels, which were slightly elevated above normal, did not improve in our study participants after the 2 follow-up periods. On the other hand, in Bagwell et al. study [15], glycated hemoglobin (HbA1c) significantly decreased after 6 months only (mean difference $0.95 \%, p=$ 0.002 ). Such results could be due to the intensive counseling along with active oral antidiabetics and insulin adjustments done by pharmacists as reported by Bagwell et al. [15].

The baseline lipid profiles of the participants that reflect a high prevalence of impaired lipid profile were comparable to those reported by Al-Nooh et al. [32], where 44.7\% had high TG levels, $47.6 \%$ had low HDL levels, and $10.8 \%$ had high LDL levels. However, we found significant improvements at both follow-up intervals in the lipid profile of the study participants even though they did not reach desirable levels. Significant reduction in LDL levels was also achieved in a study by Tsuyuki et al. [14] in 3 months only in the pharmacist care intervention group, while no significant LDL level reduction was achieved in Bagwell et al. [15] after 6 months of follow-up.

Concerning awareness of CVD risk factors, our study revealed a relatively good knowledge of these factors where the baseline awareness score was 5.67 out of 8 . It significantly improved after effective patient education at 1 - and 2-year intervals to 6.56 and 6.76 respectively. Our participants' awareness of CVDs risk factors was better than the findings of a Jordanian study [33] that reported a limited public knowledge of CVD risk factors.

The mean ASCVD risk in our study was 11 with almost half of the studied population having moderate or high risk scores. The baseline average Framingham risk score in our participants was much higher than that calculated in the Bagwell et al. [15]. However, ASCVD risk scores decreased significantly with none of the participants having high risk score after 2 years. This is the most important finding of our study where ASCVD risk reductions were both statistically significant and clinically meaningful.

\section{Limitations}

Our study lacks a national comparison group for awareness of CVD risk factors, ASCVD risk, and the number of risk factors. International and regional studies may not reflect comparable backgrounds and consequently comparable results. In addition, possible sources of bias as 
in sampling, measurements, and information might have occurred due to the observational study design. Another limitation is that a lot of statistical testing was done in this study. Since the $p$ value of 0.05 is based on running a single statistical test on a primary outcome, some of our findings are likely to be spurious due to multiple testing.

Despite having a large sample size which is considered a strength, it resulted in statistically significant results that were not clinically meaningful.

One of our main strengths is that this is the first study in Lebanon to evaluate the role of pharmacists in improving CVDs risk factors. The added value of this study is the concomitant assessment of several CVD risk factors in one procedure. In addition, this study widened the scope from secondary to primary prevention of CVD through interventions to prevent the occurrence of full-blown diabetes, dyslipidemia, and hypertension along with active management of obesity, physical inactivity, improper diet, and smoking.

\section{Conclusion}

CVDs constitute an important public health problem that can be targeted by integrating pharmacists into the healthcare programs. Education and counseling of partici- pants done by the pharmacists involved in this study significantly emphasizes the role of pharmacists improving and modifying CVD risk factors. We strongly support the adoption of a plan that includes pharmacists as important contributors to address the rise in CVDs and their risk factors. This plan should aim to increase public and professional awareness of the burden of CVDs in Lebanon and aid in regular monitoring and follow-up.

\section{Acknowledgments}

We thank the subjects for agreeing to participate in this study.

\section{Disclosure Statement}

The authors declare that they have no conflict of interests to disclose.

\section{Author Contribution}

I.M.F. and S.H. contributed toward study design, data collection, analysis and interpretation, manuscript drafting, and writeup of the paper. M.K.R. and D.N.M. contributed toward the conception of the hypothesis and project supervision. All authors read and approved of the final manuscript.

\section{References}

1 Cardiovascular Diseases: World Health Organization (WHO). 2016. http://www.who.int/ mediacentre/factsheets/fs317/en/.

2 Global Burden Disease Profile: Lebanon. 2010. http://www.healthdata.org/.

3 Santschi V: Impact of pharmacist care in the management of cardiovascular disease risk factors. Arch Intern Med 2011;171:1441.

4 Preslaski CR, et al: Pharmacist contributions as members of the multidisciplinary ICU team. Chest 2013;144:1687-1695.

5 Odum L, Whaley-Connell A: The role of team-based care involving pharmacists to improve cardiovascular and renal outcomes. Cardiorenal Med 2012;2:243-250.

6 Taegtmeyer AB, Kullak-Ublick GA, Widmer N, Falk V, Jetter A: Clinical usefulness of electronic drug-drug interaction checking in the care of cardiovascular surgery inpatients. Cardiology 2012;123:219-222.

7 Mdege ND, Chindove S: Effectiveness of tobacco use cessation interventions delivered by pharmacy personnel: a systematic review. Res Soc Adm Pharm 2014;10:21-44.

8 Machado M, et al: Sensitivity of patient outcomes to pharmacist interventions. Part II:
Systematic review and meta-analysis in hypertension management. Ann Pharmacother 2007;41:1770-1781.

9 Lee JK, et al: Effect of a pharmacy care program on medication adherence and persistence, blood pressure, and low-density lipoprotein cholesterol: a randomized controlled trial. JAMA 2006;296:2563-2571.

10 Olson K, Potts L: Role of the pharmacist in the management of dyslipidemia. J Pharm Pract 2006;19:94-102.

11 Usherwood T: National guidelines for the management of absolute cardiovascular disease risk. Med J Aust 2013;199:243244.

12 Liu Y, et al: Community pharmacist assessment of 10-year risk of coronary heart disease for union workers and their dependents. J Am Pharm Assoc 2008;48:515-517.

13 Mc Namara KP, et al: Engaging community pharmacists in the primary prevention of cardiovascular disease: protocol for the Pharmacist Assessment of Adherence, Risk and Treatment in Cardiovascular Disease (PAART CVD) pilot study. BMC Health Serv Res 2010;10:264.
14 Tsuyuki RT, Al Hamarneh YN, Hassan I, Jones CA, Hemmelgarn BR: RxEACH: pharmacist prescribing reduces CVD risk. Pharm Economics Outcomes News 2016;751:27.

15 Bagwell A, et al: The role of clinical pharmacists in modifying cardiovascular disease Risk factors. Inov Pharm 2013;4:129.

16 Fahs I, et al: The prevalence and awareness of cardiovascular diseases risk factors among the lebanese population: a prospective study comparing urban to rural populations. Cardiol Res Prac 2017;1-10.

17 Smith SC Jr, et al: Our time: a call to save preventable death from cardiovascular disease (heart disease and stroke). J Am Coll Cardiol 2012;60:343-2348.

18 Research Randomizer: Randomizerorg. 2018. https://www.randomizer.org/.

19 EUR-Lex: 31990D0033 - EN-EUR - Lex: Eur-lexeuropaeu. 2018. http://eur-lex.europa.eu/legal-content/EN/TXT/? uri= CELEX\%3A31990D0033.

20 Preventing Cardiovascular Diseases and Heart Disease: Info Patient. 2015. http://patient.info/health/preventing-cardiovasculardiseases.
Pharmacist and Cardiovascular Risk Factors
Med Princ Pract 2018;27:508-514

DOI: $10.1159 / 000490853$ 
21 Cardiovascular disease risk factors: World Heart Federation. 2015. http://www.worldheart-federation.org/cardiovascular-health/ cardiovascular-disease-risk-factors/.

22 WHO, The STEPS Instrument and Support Materials. 2003. http://www.who.int/chp/ steps/instrument/en/.

23 CDC, Behavioral Risk Factor Surveillance System, Annual Survey Data. 2015. http:// www.cdc.gov/brfss/annual_data/annual_ data.htm.

24 Guidelines for Data Processing and Analysis of the International Physical Activity Questionnaire. 2002. http://www.institutferran.org/ documentos/Scoring_short_ipaq_april04.pdf.

25 Food Frequency Questionnaire. 2003. https:// wwwn.cdc.gov/Nchs/Nhanes/2003-2004/ FFQDC_C.htm.

26 Adult Questionnaire (18-64): Outcomes Measurement System (OMS). 2009. http:// maryland.beaconhealthoptions.com/ provider/forms/oms/OMS_Adult_Full_ Qx.pdf.

27 Wagner J, et al: Development of a questionnaire to measure heart disease risk knowledge in people with diabetes: The Heart Disease Fact Questionnaire. Patient Educ Couns 2005;58:82-87.

28 CDC, NHANES Anthropometry and Physical Activity Monitor Procedures Manual. 2005. https://www.cdc.gov/nchs/data/ nhanes/nhanes_05_06/BM.pdf.

29 Expert Panel on Detection, Evaluation, and Treatment of High Blood Pressure: The Seventh Report of the Joint National Committee on Prevention, Detection, Evaluation, and Treatment of High Blood Pressure: the JNC 7 Report. JAMA 2003;290:197.

30 Expert Panel on Detection, Evaluation, and Treatment of High Blood Cholesterol in
Adults: Executive Summary of the Third Report of the National Cholesterol Education Program (NCEP) Expert Panel on Detection, Evaluation, and Treatment of High Blood Cholesterol in Adults (Adult Treatment Panel III). JAMA 2001;285:2486-2497.

31 Yamout R, et al: Screening for cardiovascular risk in asymptomatic users of the primary health care network in Lebanon, 2012-2013. Prev Chronic Dis 2014;11.

32 Al-Nooh AA, et al: The prevalence of cardiovascular disease risk factors among employees in the Kingdom of Bahrain between October 2010 and March 2011: a cross-sectional study from a workplace health Campaign. Cardiol Res Pract 2014;2014:1-9.

33 Mukattash TL, et al: Public knowledge and awareness of cardiovascular disease and its risk factors: a cross-sectional study of 1,000 Jordanians. Int J Pharm Pract 2012;20:367. 